\title{
Effect of wavy tool path on the stability properties of milling by the implicit subspace iteration method
}

Mate TOTH

Department of Applied Mechanics, Budapest University of Technology and Economics Muegyetem rkp 3, Budapest H-1111 HUNGARY

toth_m@mm.bme.hu

Daniel BACHRATHY ${ }^{1}$

Department of Applied Mechanics, Budapest University of Technology and Economics Muegyetem rkp 3, Budapest H-1111 HUNGARY

bachrathy@mm.bme.hu

Gabor STEPAN

Department of Applied Mechanics, Budapest University of Technology and Economics Muegyetem rkp 3, Budapest H-1111 HUNGARY

stepan@mm.bme.hu

${ }^{1}$ Corresponding author: Daniel BACHRATHY, bachrathy@mm.bme.hu 


\begin{abstract}
There are several practical methods to reduce machine tool vibrations that have negative effects especially on the quality of the machined surface. The most intricate vibration is the regenerative one originated in a delay effect of cutting processes. One group of the methods that may be successful in avoiding regenerative vibrations is the appropriate variation of the corresponding time delay. This study presents the stability analysis of milling processes in case of an especially intricate way of varying the delay in time: the radial depth of cut is varied in face milling resulting in a wavy tool path. The combination of the semidiscretization method and the implicit subspace iteration method is introduced to present an efficient way of calculating stability charts that provide conclusions regarding the use of this method in eliminating chatter.
\end{abstract}

Keywords: chatter; wavy tool path; implicit subspace iteration; semi-discretization

\title{
1. Introduction
}

Machine tool chatter has a negative effect on the lifetime of the machine tools and on the quality of the surface finish of the workpiece [1-4]. This self-excited vibration is originated in the so-called surface regeneration effect [3-5]. The mathematical modeling of chatter is rooted in the theory of delay differential equations (DDEs) [6], and the primary cause of instability is the presence of a large time delay in the cutting processes.

A constant single point delay appears in the DDE of the commonly used mechanical models of milling processes at constant spindle speed if conventional helical tools with even pitch angles are used. The time delays are proportional to the pitch angles between two neighboring cutting edges, and inversely proportional to the spindle speed.

Different tool geometries and operating conditions have been developed in order to improve the stability properties of cutting processes by weakening the negative effect of the inherent time delay by disturbing the concentrated nature of the delayed term in the corresponding equations of motion.

In case of milling processes, the concentrated nature of the time delay can be changed to a more disturbed one by introducing uneven pitch angles between the cutting edges $[7,8,9,10]$. This idea results in multiple discrete time delays in the system, whose number depends on the number of teeth of the tool and the applied pitch angle distribution $[11,12,13]$.

Milling tools with varying helix angle $[14,15,16,17,10]$ or with wavy cutting edges $[18,19]$ are used to achieve a similar effect by distributing the concentrated time delay even further to continuously varying delays over a given delay-interval. In these cases, the pitch angles are changing continuously along the axial coordinate of the tool, which leads to DDE models with distributed time delays [20]. In addition to the continuously varying 
helix angle, wavy cutting edge profiles give the opportunity to modify/design the weight function of the distributed delay terms in the governing equations [5,21,22].

Serrated tools $[23,24,10]$ are also designed to perturb the regenerative phase shift. Serration profiles have phase shift from one cutting edge to the subsequent one. The geometry of serration causes the actual machining edge to cut the surface marks of different previous teeth along the axial direction of the tool. The result is the appearance of multiple discrete delays which depend on the applied serration density and on the actual feed per tooth.

Applying varying spindle speed $[25,26,27,28,29]$ to the cutting process also affects the time delay of the system directly. In case of turning [20], the periodic spindle speed variation results in a continuously changing time delay, which periodically varies the otherwise constant delay over a specific interval determined by the range of the spindle speed variation. The same applies to milling operations with continuously varying spindle speed, however, this leads to DDE models with time periodic parameters both in the cutting coefficients and in the delays.

Note, that all the above mentioned methods create an uneven distribution of the cutting force between or along the cutting edges, which could lead to decreased tool life. Moreover, in the case of spindle speed variation, the cutting load fluctuates in time which also leads to increased tool wear [29].

Considering the applications above aiming to improve cutting stability by perturbing the time delay itself and thus suppress the regenerative effect, a similar possible way to decrease the appearance of chatter might be the variation of the coefficient of the delayed term in the DDE model instead of varying the time delay itself. In this case, the cutting conditions could be kept at an optimal level, which would not shorten the tool life. This can be achieved, for example, by means of a wavy tool path that causes the variation of the entering and exiting angles of the cutting edges periodically, therefore, it causes an additional periodic variation of the time periodic coefficient of the delayed term in the DDE model. Actually, it leads to a quasi-periodic parametric excitation, since the corresponding coefficient of the delayed term is already periodic with the time delay, which is perturbed now further periodically with the wavy path having an independent time period. Since it leads to an uneven surface finish, the wavy tool path can primarily be used in roughing processes, similarly to the application of serrated tools.

The present study investigates the stability of milling processes in the latter case. This requires the improved application of the semi-discretization method (SDM) [30,31,32] combined with the implicit subspace iteration method (ISIM) $[33,34]$. The structure of the paper is as follows. First, the simplified mechanical model of milling is presented and the corresponding mathematical model is derived in case of wavy tool paths. Then SDM and ISIM are implemented with special attention to the computation time required for the construction of stability charts in the parameter plane of spindle speed and axial depth of 
cut. The resulting stability charts then provide conclusions regarding the effectiveness of applying wavy tool paths in order to reduce the probability of chatter.

\section{Mechanical model of milling}

In order to analyze the effect of a wavy tool path on the stability properties of milling, a single degree-of-freedom (DoF) model is considered in Fig. 1. The tool is assumed to be flexible with a single relevant vibration mode in the feed direction, and the workpiece is considered to be rigid. The corresponding equation of motion reads

$$
\ddot{x}(t)+2 \zeta \omega_{\mathrm{n}} \dot{x}(t)+\omega_{\mathrm{n}}^{2} x(t)=\frac{1}{m} F_{x}(t),
$$

where $\omega_{\mathrm{n}}$ is the natural angular frequency of the tool, $\zeta$ is the corresponding damping ratio, $m$ denotes the effective modal mass, and $F_{x}$ is the $x$ component of the resultant cutting force acting on the tool. Figure 2 presents an up-milling operation with spindle speed $\Omega$ given in $[\mathrm{rad} / \mathrm{s}]$.

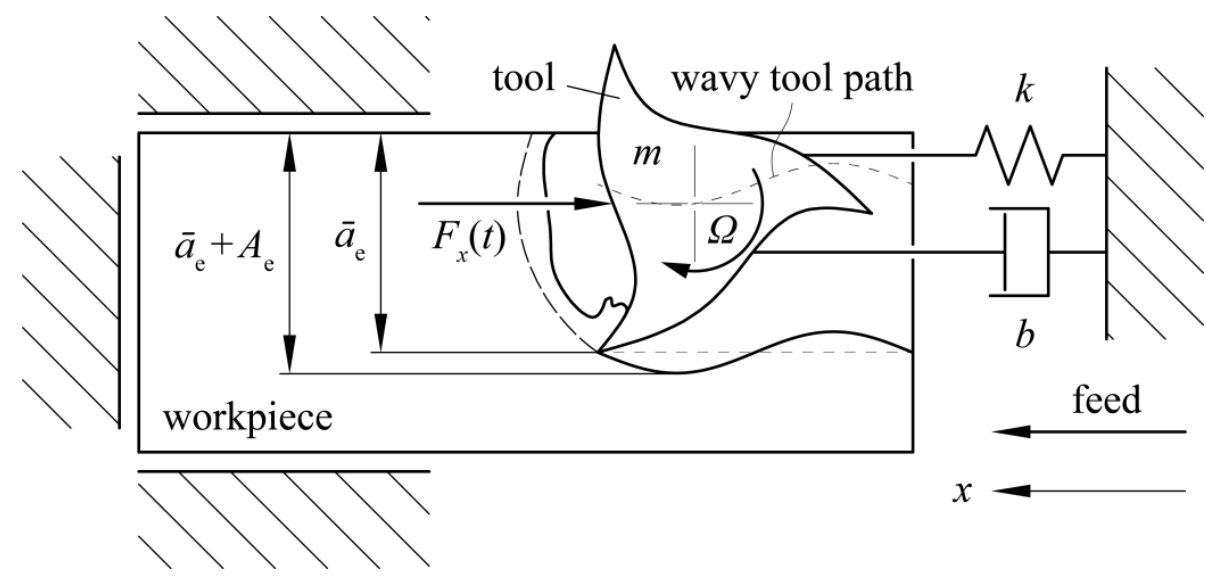

Fig. 1: Mechanical model of milling with a wavy tool path

The wavy tool path is generated by means of the harmonic variation of the radial depth of cut, which assumes the form

$$
a_{\mathrm{e}}(t)=\bar{a}_{\mathrm{e}}+A_{\mathrm{e}} \cos \left(\frac{2 \pi t}{T}\right),
$$

where $\bar{a}_{e}$ is the average radial depth of cut and $A_{\mathrm{e}}$ is the amplitude of the wavy tool path. The time needed for the tool to complete one period of the depth of cut variation is denoted by $T$. Due to the wavy tool path, the entering angles $\varphi_{\text {en }}$ and the exiting angles $\varphi_{\text {ex }}$ of the cutting teeth change according to the radial depth of cut function. As it can be checked with the help of Fig. 2, these angles are now time-dependent and can be calculated for up-milling and down-milling as 


$$
\begin{gathered}
\varphi_{\mathrm{en}}^{\mathrm{up}}(t)=\arctan \left(-\frac{\dot{a}_{\mathrm{e}}(t)}{D}\right), \quad \varphi_{\mathrm{en}}^{\mathrm{down}}(t)=\arccos \left(2 \frac{a_{\mathrm{e}}(t)}{D}-1\right), \\
\varphi_{\mathrm{ex}}^{\mathrm{up}}(t)=\arccos \left(1-2 \frac{a_{\mathrm{e}}(t)}{D}\right), \quad \varphi_{\mathrm{en}}^{\text {down }}(t)=\arctan \left(\frac{\dot{a}_{\mathrm{e}}(t)}{D}\right),
\end{gathered}
$$

respectively, where $D$ is the diameter of the tool.

A straight-fluted tool is considered (helix angle $\eta=0$ ) with number $N$ of cutting teeth. The tangential and radial cutting force components acting on tooth $j$ have the forms

$$
\begin{aligned}
& F_{j, \mathrm{t}}(t)=a_{\mathrm{p}} g_{j}(t) K_{\mathrm{t}} h_{j}^{q}(t), \\
& F_{j, \mathrm{r}}(t)=a_{\mathrm{p}} g_{j}(t) K_{\mathrm{r}} h_{j}^{q}(t),
\end{aligned}
$$

where $a_{\mathrm{p}}$ is the axial depth of cut, $K_{\mathrm{t}}$ and $K_{\mathrm{r}}$ are the tangential and radial cutting force coefficients, respectively [2], $h_{j}$ is the actual chip thickness cut by tooth $j, q$ is the cutting force exponent, and $g_{j}$ is a screen function determining whether tooth $j$ is in $\left(g_{j}=1\right)$ or out $\left(g_{j}=0\right)$ of the cut [35]. The actual chip thickness cut by tooth $j$ can be obtained as

$$
h_{j}(t)=\left(f_{\mathrm{z}}+x(t-\tau)-x(t)\right) \sin (\tilde{\varphi}(t)),
$$

where $f_{\mathrm{z}}$ is the constant feed per tooth. The regenerative effect is described by $x(t-\tau)$ and $x(t)$, which denote the delayed and actual positions of the tool, respectively [5]. The instantaneous angular position of tooth $j$ is represented by $\varphi_{j}$, and the time delay can be calculated as $\tau=2 \pi / N / \Omega$ if the cutting edges are uniformly distributed. The angle between the overall and instantaneous feeds is represented by $\tilde{\varphi}(t)$ which is equal to $\varphi_{\text {en }}(t)$ for upmilling and $\pi-\varphi_{\mathrm{ex}}(t)$ for down-milling. This term is necessary for defining the angular position which corresponds to zero chip thickness.
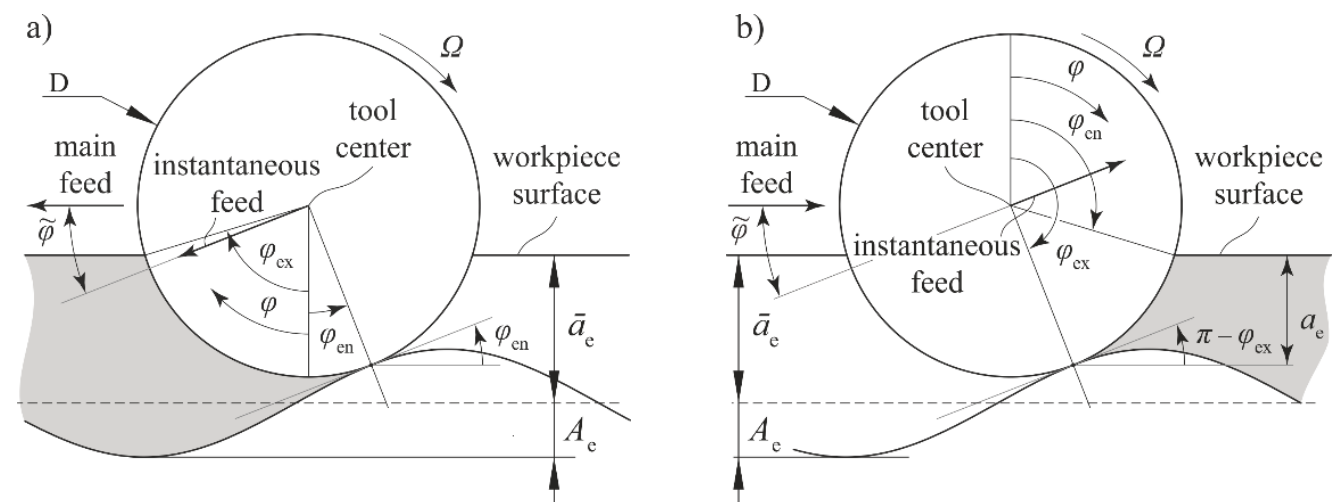

Fig. 2: Entering and exiting angles for up (a) and down-milling (b). Grey areas represent the material to be removed.

Substituting (3), (4) and (5) back to the equation of motion (1), the general solution has a periodic part $x_{\mathrm{p}}(t)$ and its variation $\xi(t)$ which can be interpreted as the perturbation of the stationary milling process described by the particular solution $x_{\mathrm{p}}(t)$. Substituting the 
general solution $x(t)=x_{\mathrm{p}}(t)+\xi(t)$ back to Eq. (1), and linearizing around the trivial solution of the perturbation parameter, the equation of motion has the form

$$
\ddot{\xi}(t)+2 \zeta \omega_{\mathrm{n}} \dot{\xi}(t)+\left(\omega_{\mathrm{n}}^{2}+G\left(t, a_{\mathrm{p}}\right)\right) \xi(t)=G\left(t, a_{\mathrm{p}}\right) \xi(t-\tau),
$$

where $G\left(t, a_{\mathrm{p}}\right)$ is a particular directional factor [35, 36, 37], that depends on the axial depth of cut linearly, and it is a quasi-periodic function of time $t$. In case of a straight tool path, it is simply periodic with the tooth passing period $\tau$ of the system. However, for a wavy tool path, this period is modulated by the time period $T$ of the sinusoidal depth of cut. If $T$ $/ \tau$ is rational then $G\left(t, a_{\mathrm{p}}\right)$ remains periodic with a possibly large principal period, otherwise it is quasi-periodic. For practically realizable sinusoidal depth of cut functions $T \gg \tau$ holds and it is also reasonable to assume those cases only when $T$ is simply an integer multiple of $\tau$.

The effects of different wavy tool paths on $G$ are presented in Fig. 3 for a two-fluted tool that takes five complete rotations over the time period $T$. Consequently, ten peaks of different heights, widths and shapes can be seen in each period, depending on the level of waviness applied.

In the subsequent sections, those mathematical tools are presented which are used for the stability analysis of the above systems having the above described periodic parametric excitation that is close to quasi-periodic.
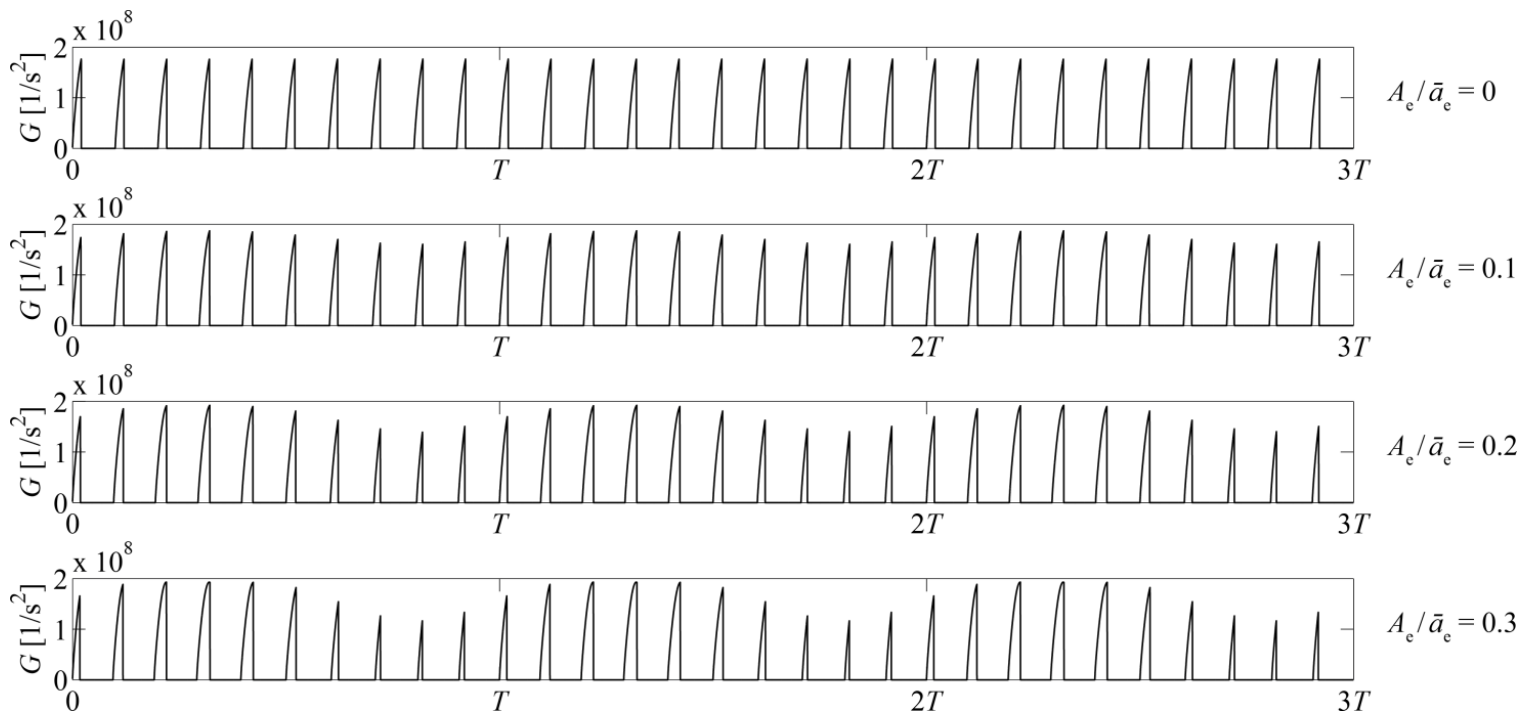

Fig. 3: Effect of a wavy tool path on the particular directional factor $G$ : straight tool path in panel a), slight waviness in panel b), moderate waviness in panel c), and severe waviness in panel d). Parameters presents in Table 1.

\section{Brief summary of the semi-discretization method}


There have been many algorithms [30,38,39,40] developed during the last 15 years for systems where the time-delay effect is coupled with parametric excitation. The semidiscretization method (SDM) is one of the efficient ways to analyze stability $[20,41,42,43,30,31,32]$. The basic idea of the SDM is the numerical discretization of the delayed terms only above the delay-time interval. Consequently, the governing DDE becomes an ordinary differential equation (ODE) that can be solved in closed form in linear cases for each time step within these discrete delay-intervals, and a linear discrete map is constructed that describes the connection of the discretized state as a large vector over the discrete time instants within the delay interval. The size of this mapping depends on the resolution of the delay discretization.

If explicit time-periodicity also appears due to the parametric excitation, the timeperiodic coefficients (or even the delays) should also be discretized in time over the time period, and the above procedure leads to different linear mappings at each sampled instant of the time period:

$$
\mathbf{z}_{i+1}=\mathbf{G}_{i} \mathbf{z}_{i}
$$

can be defined between two states corresponding to two neighboring time steps. Here, $\mathbf{G}_{i}$ denotes the coefficient matrix connecting states $\mathbf{z}_{i}$ and $\mathbf{z}_{i+1}$, which are the vectors of the discretized states sampled at the discrete time intervals at subsequent time instants. If these linear mappings are chained by simple multiplications over the time-periodicity, a discrete map can be defined between the initial delay-discrete state $\mathbf{z}_{0}$ and the one $\mathbf{z}_{T}$ a principal period later:

$$
\mathbf{z}_{T}=\mathbf{G}_{n} \ldots \mathbf{G}_{2} \mathbf{G}_{1} \mathbf{G}_{0} \mathbf{z}_{0}=\boldsymbol{\Phi} \mathbf{z}_{0},
$$

where the transition matrix $\boldsymbol{\Phi}$ is a finite dimensional approximation of the infinite dimensional monodromy operator [44]. Thus, the stability analysis is reduced to the problem whether the absolute values of all the eigenvalues of $\boldsymbol{\Phi}$ are less than one: $\left|\mu_{i}\right|<1$. In order to improve numerical accuracy, the delay resolution, the order of semidiscretization, and the time periodicity resolution can be increased.

In the basic models of milling with constant parameters, the time delay and the time periodicity are just equal to each other and it is reasonable to apply the same resolution for both of them. This is not true for time-varying spindle speeds, or for the case in question when the tool path is wavy. In this case, certain parts of the stability boundaries require significantly higher delay resolutions than others. To resolve this problem and to achieve relevant reduction in computation time, the SDM is combined with the implicit subspace iteration method (ISIM) $[33,34]$ as explained in the next section.

\section{Implicit subspace iteration}

Consider the general eigenvalue problem 


$$
\mathbf{\Phi S}=\mathbf{S} \boldsymbol{\mu},
$$

where $\boldsymbol{\Phi}$ is an $n \times n$ square matrix, $\boldsymbol{\mu}$ is a diagonal matrix of size $n \times n$ containing the eigenvalues of $\boldsymbol{\Phi}$ on its main diagonal, and matrix $\mathbf{S}$ of size $n \times n$ consists of the eigenvectors of $\boldsymbol{\Phi}$ in its columns. A set of $N_{\mathrm{s}} \leq n$ dominant eigenvectors corresponding to the first $N_{\mathrm{s}}$ eigenvalues of the largest absolute values can be approximated in an iterative way. Let $\mathbf{S}_{j}$ of size $n \times N_{\mathrm{s}}$ denote the matrix of the $N_{\mathrm{s}}$ dominant eigenvectors after the $j^{\text {th }}$ iteration step. Taking an additional iteration step, a new set $\mathbf{V}_{j}$ of size $n \times N_{\text {s }}$ can be calculated according to

$$
\mathbf{V}_{j}=\mathbf{\Phi S}_{j}
$$

If $\mathbf{S}_{j}$ is obtained after a sufficient number of iteration steps, it will converge to the dominant eigenvectors, and the basis formed by the column vectors in both $\mathbf{S}_{j}$ and $\mathbf{V}_{j}$ span approximately the same space. Therefore, an approximate matrix $\mathbf{H}_{j}$ of size $N_{\mathrm{s}} \times N_{\mathrm{s}}$ connecting $\mathbf{S}_{j}$ and $\mathbf{V}_{j}$ can be obtained using a pseudo-inverse calculation from the relation

$$
\mathbf{V}_{j} \approx \mathbf{S}_{j} \mathbf{H}_{j} \rightarrow \mathbf{H}_{j}=\left(\mathbf{S}_{j}^{\mathrm{T}} \mathbf{S}_{j}\right)^{-1} \mathbf{S}_{j}^{\mathrm{T}} \mathbf{V}_{j}
$$

where matrix $\mathbf{H}_{j}$ represents a subspace of size $N_{\mathrm{s}} \times N_{\mathrm{s}}$ of the original space of matrix $\boldsymbol{\Phi}$ of size $n \times n$. After several iteration steps, the eigenvalues of $\mathbf{H}_{j}$ provide a good approximation for the dominant eigenvalues of $\boldsymbol{\Phi}$. This way, it is enough to compute the eigenvalues of a significantly reduced $N_{\mathrm{s}}$-sized matrix $\mathbf{H}_{j}$ instead of the large $n$-sized matrix $\boldsymbol{\Phi}$. The details of this iteration process can be found in $[33,34]$.

In the case of a known matrix $\boldsymbol{\Phi}$, the iteration can be initiated from a random array of $\mathbf{S}_{0}$, and Eqs. (10) and (11) can be used to calculate the approximation of the dominant eigenvectors after one iteration step. Normalizing the result: $\mathbf{S}_{j+1}=\mathbf{A}_{j} \mathbf{V}_{j}$ (where $\mathbf{A}_{j}$ is a matrix containing the eigenvectors of $\mathbf{H}_{j}$ ), the same formula can be applied again and again until convergence of the dominant eigenvectors is achieved.

However, it is also possible to determine the matrix $\mathbf{H}_{j}$ without calculating the whole matrix $\boldsymbol{\Phi}$, which is computationally demanding [20,45]. The vector $\mathbf{V}_{j}$ can be calculated directly from the equations of motion of the given system, starting from a random set of initial conditions $\mathbf{S}_{0}$. In this case, matrix $\mathbf{H}_{j}$ can be approximated in each iteration step until convergence is reached. In stability calculations, convergence is evaluated simply by means of the magnitude of the largest eigenvalue of $\boldsymbol{\Phi}$. Thus, the advantage of implementing the ISIM is that the transition matrix $\boldsymbol{\Phi}$ does not have to be calculated. Given an arbitrary initial set $\mathbf{S}_{0}$, the new set $\mathbf{V}_{j}$ can be obtained by time integration utilizing the linear equations of motion of the system. During the time integration process, the corresponding elements of the matrix $\mathbf{G}$ in Eq. (12) of the SDM are used, for which a closed form solution is given in [20]. Consequently, the subspace $\mathbf{H}_{j}$ can be determined iteratively 
without calculating the transition matrix for each parameter combination which is timeconsuming, especially in case of fine delay resolutions.

Figure 4 presents the computation time required to determine the stability properties of a single parameter combination against the delay resolution. The relations can be described by power functions, and the combined ISIM-SDM is significantly more efficient than the SDM alone, especially for fine delay resolutions. The computation time of the SDM with direct matrix multiplication [20] increases according to a power of 3.7, while the computation time with the combined ISIM increases only linearly when the accuracy is the same. Note, that the memory requirements of the ISIM-SDM are also better.

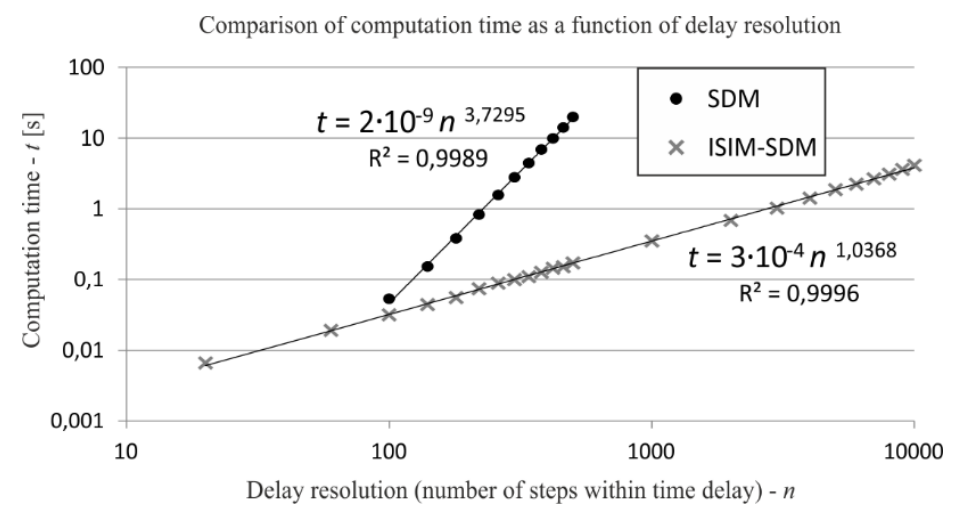

Fig. 4: Comparison of the computation time of the SDM and the ISIM-SDM as a function of delay resolution on a logarithmic scale

\section{Stability analysis}

The time period $T$ of the harmonic radial depth of cut function is defined in such a way that the tool is able to make an integer number $N_{\mathrm{r}}$ of revolutions over that time period $\left(N_{\mathrm{r}}=T / \tau / N\right)$. This idea is used for preserving the simplicity of the analysis without losing any possible effect of the wavy tool path. The stability charts in Figures 5 and 7 present the effects of both the amplitude and the time period of the harmonic depth of cut function.

In these case studies, the single relevant natural frequency of the tool is considered to be $400 \mathrm{~Hz}$, and all the other tool, cutting and process parameters are taken from a real case study in [54]. An up-milling operation is assumed with a straight-fluted end mill of two cutting edges.

\begin{tabular}{|c|c|c|c|c|c|}
\hline \multicolumn{2}{|c|}{ Tool } & \multicolumn{2}{c|}{ Modal parameters } & \multicolumn{2}{c|}{ Process parameters } \\
\hline diameter $-D$ & $20 \mathrm{~mm}$ & natural frequency $-\omega_{\mathrm{n}}$ & $400 \mathrm{~Hz}$ & average radial depth $-a_{\mathrm{e}}$ & up milling: $2 \mathrm{~mm}$ \\
\hline $\begin{array}{c}\text { No. of teeth }- \\
N\end{array}$ & 2 & modal mass $-m$ & $3.166 \mathrm{~kg}$ & cutting force exponent $-q$ & 0.75 \\
\hline helix angle $-\eta$ & $0^{\circ}$ & damping ratio $-\zeta$ & $2 \%$ & feed per tooth $-f_{\mathrm{z}}$ & $0.1 \mathrm{~mm}$ \\
\hline & & modal stiffness $-k$ & $20 \mathrm{MN} / \mathrm{m}$ & tangential cut. coeff. $-K_{\mathrm{t}}$ & $107 \mathrm{MN} / \mathrm{m}^{q+1}$ \\
\hline
\end{tabular}




\begin{tabular}{|l|l|l|l|l|l|}
\hline & & & & radial cut. coeff. $-K_{\mathrm{r}}$ & $40 \mathrm{MN} / \mathrm{m}^{q+1}$ \\
\hline
\end{tabular}

Table. 1: Parameters of the case-study

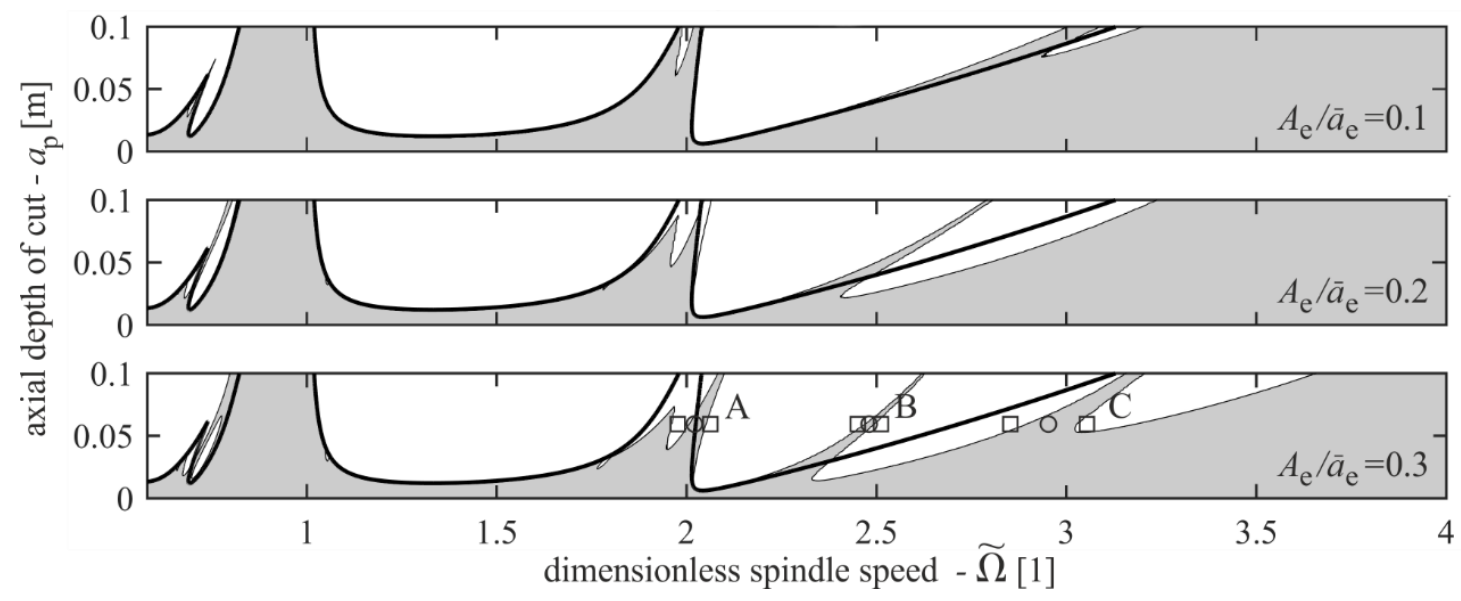

Fig. 5: Effect of the amplitude of the wavy tool path for $N_{\mathrm{r}}=5$ (shaded region), thick lines indicate the stability boundaries corresponding to a straight tool path.

The numerical simulations are performed at parameter points near to $\mathrm{A}, \mathrm{B}$ and $\mathrm{C}$; the stable and unstable motions are denoted by circles and squares, respectively.

Figure 5 shows the effect of the amplitude of the applied waviness with fixed $N_{\mathrm{r}}$ values. It can be seen that additional flip lobes appear in the stability charts as the amplitude of the radial depth cut variation gets higher. The original flip lobe below the dimensionless spindle speed coordinate $\tilde{\Omega}=N \Omega / \omega_{\mathrm{n}}=1$, which is present for a straight tool path as well, even gets detached as a flip island as the waviness of the tool path is increased (see also [46] where it was shown analytically that the flip lobes are actually islands).

In what follows, numerical simulations are presented to validate the stability properties $[47,48]$, in which the forced vibration is also considered. Simulations are performed at three points (stable, unstable, stable) around each stability boundary denoted by A, B and $\mathrm{C}$ in Fig. 5 and the corresponding resultant motions are presented by grey lines in Fig. 6. The tendencies in the simulation results validate the stability properties. For the technological parameters selected from the white area, the motions are unstable (see squares in Fig. 5) and the tool position signals start increasing exponentially. For the parameter points selected from the shaded area, the tool center motion tends to the stationary vibration caused by the periodic forcing; these are stable cases, no chatter occurs.

A simple model of the fly-over-effect is also considered to approximate the amplitude of the chatter vibration $[49,50]$. In this model, the cutting force is switched off for negative chip thickness $h_{j}(t)<0$ (see Eq.(5)). This leads to saturation in the exponentially increasing unstable vibration (see black lines in Fig. 6). The results in Fig. 6 in row A $(\tilde{\Omega}=$ 
1.98) and row $\mathrm{B}(\tilde{\Omega}=2.85 ; 3.05)$ show that if the milling process without the wavy tool path is stable (see the area below the thick line in Fig. 5), then the amplitude of the chatter vibration is significantly smaller. However, it still leads to high load on the tool.
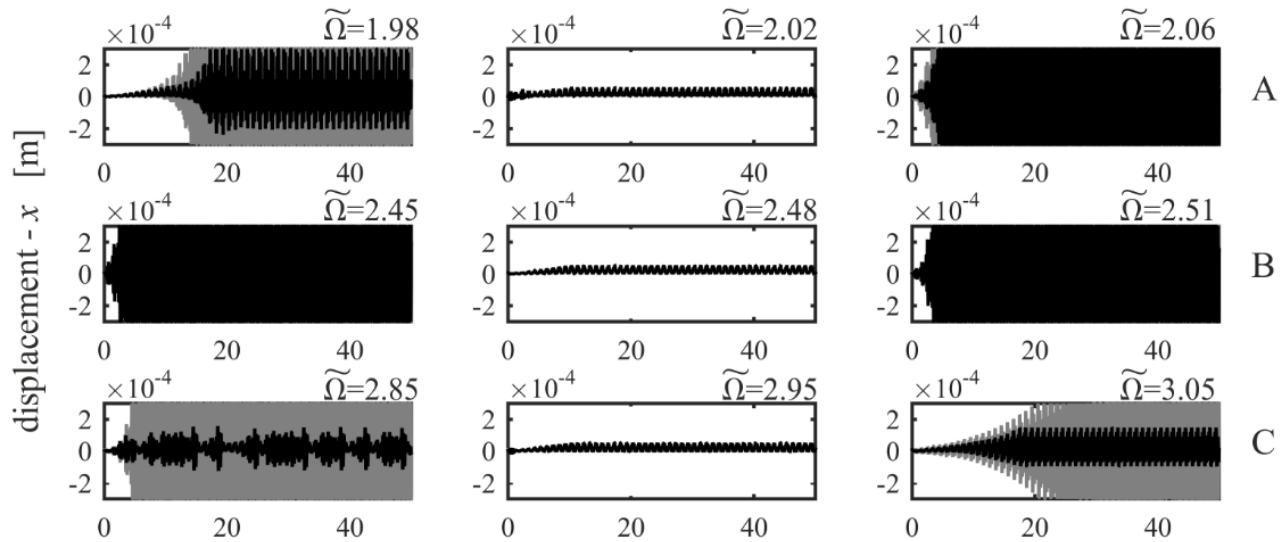

number of periods $\mathrm{t} / \mathrm{T}[-]$

Fig. 6: Numerical simulations of the tool center position $x$ for milling with harmonically varied radial depth of cut. The black and grey lines refer to simulations with and without the fly-over-effect, respectively. The middle column represents the stable processes based on Fig.5, where the stable and unstable parameters are denoted by circles and squares, respectively.

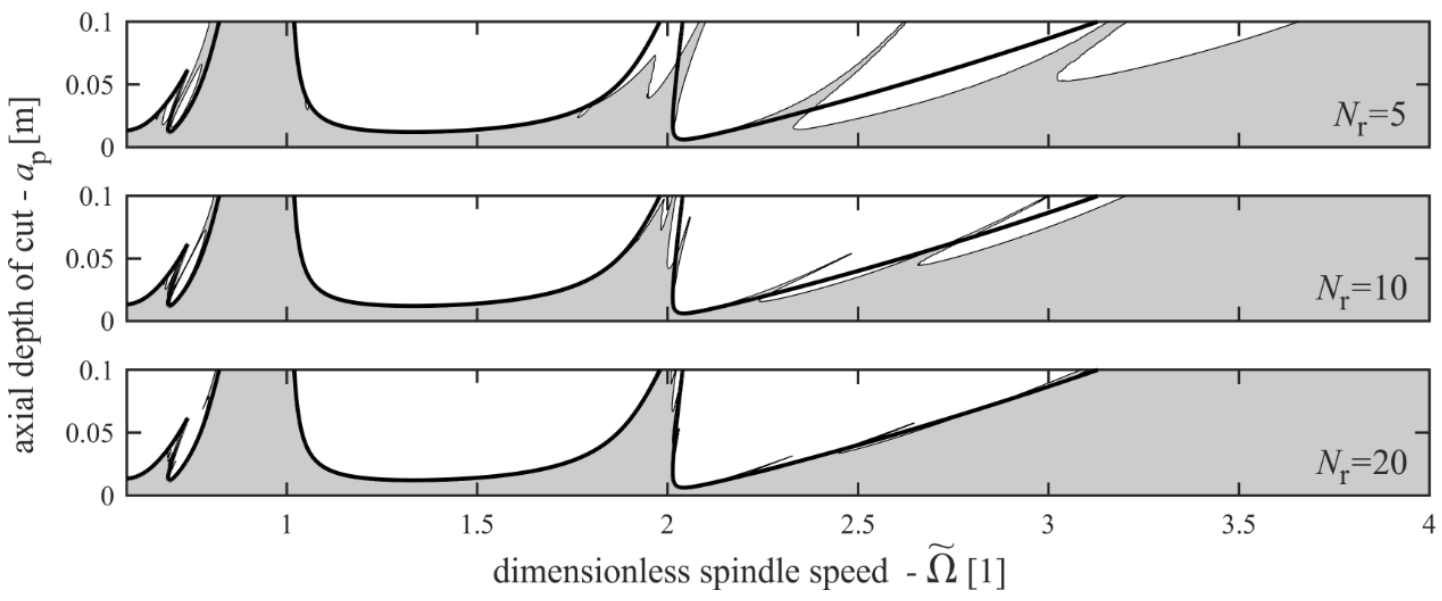

Fig. 7: Effect of the time period of the wavy tool path for $A_{\mathrm{e}} / \bar{a}_{\mathrm{e}}=0.3$ (shaded region), thick lines indicate the stability boundaries corresponding to a straight tool path

Figure 7 presents the effect of the time period for constant waviness amplitude of $A_{\mathrm{e}} / \bar{a}_{\mathrm{e}}=0.3$. As the time period increases, the stability charts corresponding to a harmonic radial depth of cut variation get closer to that of the straight tool path. Practically realistic time periods correspond to several hundreds of revolution over one harmonic radial depth of cut variation $\left(N_{\mathrm{r}}>>100\right)$. Still, the convergence of the stability chart is so fast to the chart of the straight tool path that it does not seem to be reasonable to do calculations with larger 
values of $N_{\mathrm{r}}$, since the calculation time would be several days even with the combined ISIM-SDM. As a conclusion we can state that the effect of the realistic time periods is not relevant, even for tool path with large waviness amplitude.

Note, that while the $N_{\mathrm{r}}=1$ case is not realistic as a wavy tool path, it can still be used as a simplified model of milling tool with runout $[51,52,53,54]$ while the tool path is still straight. In this case, the edges cut different radial depths, hence, the corresponding directional factor $G$ varies within one period, similarly to the $N_{\mathrm{r}}=1$ case with wavy tool path without runout. Figure 8 presents a series of stability charts corresponding to $N_{\mathrm{r}}=1$ and $A_{\mathrm{e}} / \bar{a}_{\mathrm{e}}=0.1$ for different number of edges.

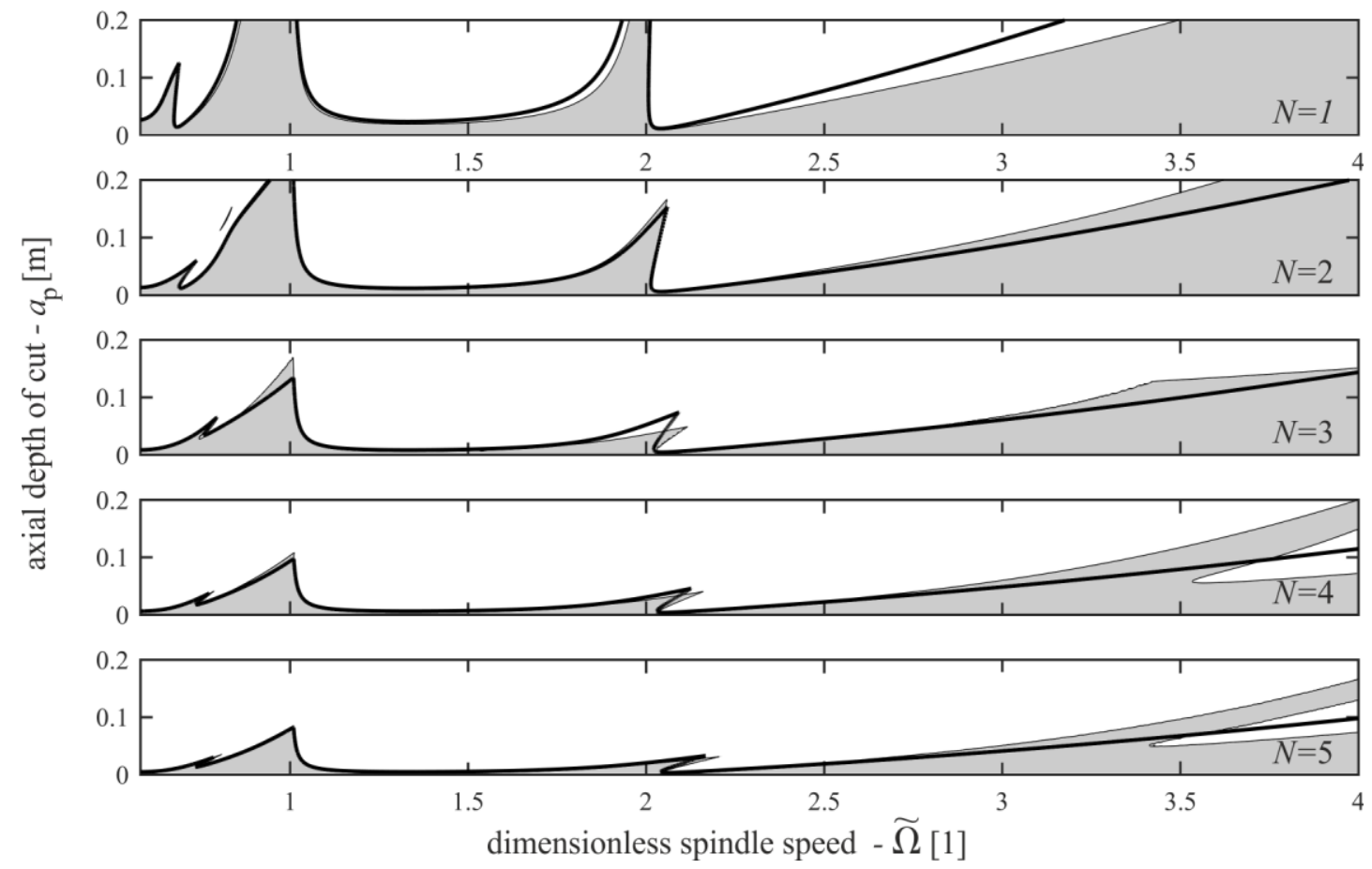

Fig. 8: Effect of the number of cutting edges for $N_{\mathrm{r}}=1$ and $A_{\mathrm{e}} / \bar{a}_{\mathrm{e}}=0.1$ (shaded region), thick lines indicate the stability boundaries corresponding to a straight tool path

Figure 8 shows that even such a simple model of machine tool runout can predict detectable effects of this phenomenon on the stability charts. The amount of runout modeled by a waviness amplitude of $A_{\mathrm{e}} / \bar{a}_{\mathrm{e}}=0.1$ constitutes a physically possible scenario, however, it cannot be considered as a negligible eccentricity. For example, in the case of two cutting edges, it means that one tooth cuts $10 \%$ more and the other $10 \%$ less compared to the ideal runout-free tool.

The case of $N=2$ cutting edges is identical to the case investigated by Insperger et al. in [54] where the difference between the stability boundaries corresponding to zero and nonzero runout is infinitesimal. It can be seen in Fig. 8 that the stability boundaries 
corresponding to the straight and wavy tool paths are close to each other, however, the very high speed region presents significant differences. It is important to note that such high cutting speeds are not considered in [54], in fact, the highest displayed dimensionless spindle speed in [54] is less than $\tilde{\Omega}=1$. Therefore, it can be concluded that the comparable parts of the stability boundaries at low spindle speeds in Fig. 9 of this study and those in [54] practically coincide for zero and nonzero runout. Also, the maximum applicable spindle speed is inversely proportional to the amount of runout of the machine tool, therefore, the stability boundaries corresponding to very high spindle speeds fall out of the scope of practical importance.

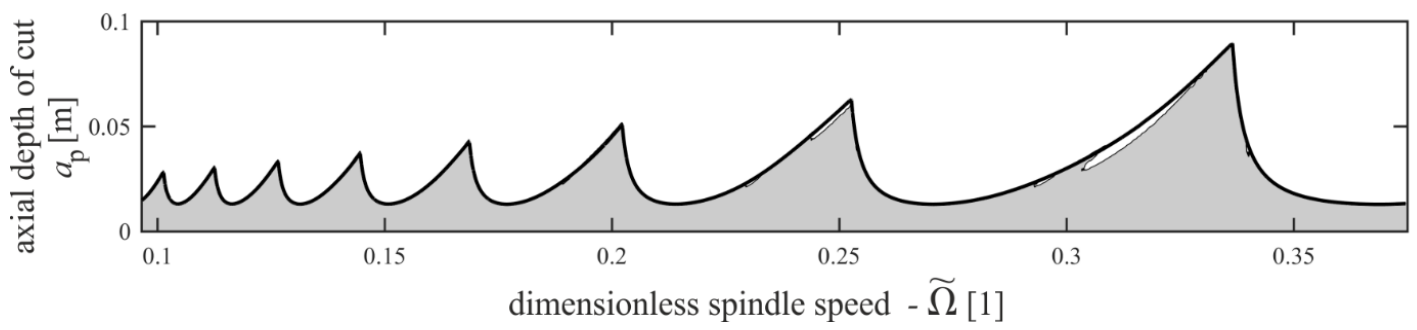

Fig. 9: Effect of a wavy tool path $\left(N_{\mathrm{r}}=5, A_{\mathrm{e}} / \bar{a}_{\mathrm{e}}=0.3\right)$ for lower spindle speeds (shaded region), the thick line indicates the stability boundaries corresponding to a straight tool path

It can be concluded from Fig. 9 that the higher the lobe number is, the less effect the wavy tool path has on the stability boundaries. This is in correspondence with the observation in [2], namely that for higher order lobes the zeroth-order approximation method provides accurate enough stability boundaries. Accordingly, the wavy tool path has small effect on stability at lower spindle speeds, since its harmonic variation can be canceled out by the time averaging of the zeroth-order approximation.

\section{Summary}

The generation of a wavy tool path in milling processes can introduce additional timeperiodicity into the delay-induced regenerative vibrations and this way, it may help to reduce the appearance of chatter similarly to many other techniques that try to mismatch the delay effects during cutting. The mathematical model of such a milling operation was derived for a simple one DoF mechanical model, and it was shown that the model typically leads to a quasi-periodic parametric excitation in the DDE model.

It was shown that the stability analysis of these systems is extremely time-consuming even if the most efficient methods (like the SDM) are used for long periodic approximations of the quasi-periodicity. The combination of the SDM with the ISIM improves the efficiency of these calculations and makes it possible to construct stability charts for these cases in reasonable time. 
The analysis of the resulting stability charts showed that the application of a wavy tool path affects the stability properties in the domain of high cutting speeds only. However, even this effect is negligible for realistic harmonic radial depth of cut amplitudes and periods: the significant impact of large amplitudes gets suppressed as the time period approaches practical values. Based on the test cases, it can be concluded that perturbing the time periodic coefficient of the delayed term only is not a successful way to weaken the negative effect of surface regeneration and thus to achieve practical improvement in the stability properties of milling operations. These results generalize the observations of Insperger et al. [54] achieved for the investigation of the effect of runout on chatter in milling processes. The parameters that look extreme at the moment could change in the future and the presented results can serve as the source of a new direction for research in fighting against chatter.

\section{Acknowledgement}

The research leading to these results has received funding from the European Research Council under the European Union's Seventh Framework Programme (FP7/2007-2013) ERC Advanced grant agreement No340889 and it was supported by the Hungarian

Scientific Research Fund - OTKA PD-112983 and the Janos Bolyai Research Scholarship of the Hungarian Academy of Sciences.

\section{References}

[1] E. Budak, Y. Altintas, Analytical Prediction of Chatter Stability in Milling - Part I: General Formulation. Journal of Dynamic Systems Measurement and Control 120 (1998) pp. 22-30

[2] Y. Altintas, Manufacturing Automation: Metal Cutting Mechanics, Machine Tool Vibrations, and CNC Design, Cambridge University Press, Cambridge, 2000

[3] J. Tlusty, Manufacturing Processes and Equipment, Prentice Hall, New Jersey, 2000

[4] S. A. Tobias, Machine Tool Vibration, Blackie and Son, London, 1965

[5] G. Stepan, Retarded Dynamical Systems, Longman, Harlow, 1989

[6] J. Hale, Theory of Functional Differential Equations, Springer Verlag, New York, 1977

[7] E. Budak, An analytical design method for milling cutters with nonconstant pitch to increase stability, Part I: Theory, J. Manuf. Sci. Eng. (2003) 125(1) pp. 29-35

[8] E. Budak, An analytical design method for milling cutters with nonconstant pitch to increase stability, Part II: Application, J. Manuf. Sci. Eng. (2003) 125(1) pp. 35-38.

[9] X. Jin, Y. Sun, Q. Guo, D. Guo, 3D stability lobe considering the helix angle effect in thin-wall milling. International Journal of Advanced Manufacturing Technology, (2016) 82(9) pp. 2123-2136 
[10] Tunc LT, Ozkirimli O, Budak E, Generalized cutting force model in multi-axis milling using a new engagement boundary determination approach. International Journal of Advanced Manufacturing Technology, (2015) 77(1) pp. 341-355

[11]J. Slavicek, The effect of irregular tooth pitch on stability of milling. In: Proceedings of the 6th MTDR Conference, Pergamon Press, London (1965) pp. 15-22

[12] H. Opitz, E. U. Dregger, H. Roese, Improvement of the dynamic stability of the milling process by irregular tooth pitch, Proc. Adv. MTDR Conf. (1966) 7 pp. 213227

[13] P. Vanherck, Increasing milling machine productivity by use of cutters with nonconstant cutting edge pitch, 8th MTDR Conference, Manchester (1967) pp. 947-960

[14] B. M. Imani, M. H. Sadeghi, M. K. Nasrabadi, Effects of Helix Angle Variations on Stability of Low Immersion Milling, IUST Int. J. Eng. Sci. (2008) 19(5), pp. 115122

[15] S. Turner, D. Merdol, Y. Altintas, K. Ridgway, Modelling of the Stability of Variable Helix End Mills, Int. J. Mach. Tools Manuf. (2007) 9-47(9), pp. 1410-1416

[16] N. Sims, B. Mann, S. Huyanan, Analytical Prediction of Chatter Stability for Variable Pitch and Variable Helix Milling Tools, J. Sound Vib. (2008) 317(3-5), pp. 664-686

[17] Q. Guo1, Y. Jiang, B. Zhao, P. Ming, Chatter modeling and stability lobes predicting for non-uniform helix tools. International Journal of Advanced Manufacturing Technology, (2016) 1-16 online-first, doi=10.1007/s00170-016-8458-y

[18] Q. Song, X. Ai, J. Zhao, Design for Variable Pitch End Mills With High Milling Stability, Int. J. Adv. Manuf. Technol. (2011) 55, pp. 891-903

[19] A. R. Yusoff, N. D. Sims, Optimisation of Variable Helix Tool Geometry for Regenerative Chatter Mitigation, Int. J. Mach. Tools Manuf. (2011) 51(2), pp. 133141

[20] T. Insperger, G. Stepan, Semi-Discretization for Time-Delay Systems, Springer, New York, 2011

[21] G. Stepan, Modelling nonlinear regenerative effects in metal cutting, Phil. Trans. R. Soc. Lond. A (2001) 359, pp. 739-757

[22] Z. H. Wang, H. Y. Hu, Robust stability test for dynamic systems with short delays by using Padé approximation, Nonlinear Dynamics (1999) 18, pp. 275-287

[23] Z. Dombovari, Y. Altintas, G. Stepan, The Effect of Serration on Mechanics and Stability of Milling Cutters, Int. J. Mach. Tools Manuf. (2010) 50(6), pp. 511-520

[24] S. D. Merdol, Y. Altintas, Mechanics and Dynamics of Serrated Cylindrical and Tapered End Mills, ASME J. Manuf. Sci. Eng. (2004) 126(2), pp. 317-326

[25] T. Inamura, T. Sata, Stability analysis of cutting under varying spindle speed. Annals of the CIRP 1974 23(1), 119-120

[26] T. Insperger, G. Stepan, Stability Analysis of Turning With Periodic Spindle Speed Modulation Via Semi-discretization, J. Vib. Control (2004) 10, pp. 1835-1855 
[27] M. Zatarain, I. Bediaga, J. Munoa, R. Lizarralde, Stability of Milling Processes With Continuous Spindle Speed Variation: Analysis in the Frequency and Time Domains, and Experimental Correlation, CIRP Ann. (2008) 57(1), pp. 379-384

[28] J. Alvarez, M. Zatarain, D. Barrenetxea, N. Ortega, I. Gallego, Semi-discretization for stability analysis of in-feed cylindrical grinding with continuous workpiece speed variation. International Journal of Advanced Manufacturing Technology, (2013) 69(1), 113-120

[29] P. Albertelli, V. Mussi, M. Monno, The analysis of tool life and wear mechanisms in spindle speed variation machining. International Journal of Advanced Manufacturing Technology, (2014) 72(5), 1051-1061

[30] T. Insperger, Full-discretization and semi-discretization for milling stability prediction: Some comments, Int. J. Mach. Tool Manu. (2010) 50:658-662

[31] T. Insperger, G. Stepan, Updated semi-discretization method for periodic delay differential equations with discrete delay. Int. J. Numer. Meth. Eng. (2004) 61:117141

[32] T. Insperger, G. Stepan, J. Turi, On the higher-order semi-discretizations for periodic delayed systems. J. Sound Vib. (2008) 313:334-341

[33] M. Zatarain, Z. Dombovari, Stability analysis of milling with irregular pitch tools by the implicit subspace iteration method, Springer-Verlag, Berlin, 2014

[34] M. Zatarain, J. Alvarez, I. Bediaga, J. Munoa, Z. Dombovari, Implicit subspace iteration as an efficient method to compute milling stability lobe diagrams, SpringerVerlag, London, 2014

[35] D. Bachrathy, T. Insperger, G. Stepan, Surface properties of the machined workpiece for helical mills. Machining Science and Technology 13 (2009) 227-245

[36] Y.C. Kao, N.T. Nguyen , M.S. Chen, S.T. Su, A prediction method of cutting force coefficients with helix angle of flat-end cutter and its application in a virtual threeaxis milling simulation system. International Journal of Advanced Manufacturing Technology, (2015) 77(9) 1793-1809

[37] Z. Zhang, H. Li, G. Meng \& S. Ren, J. Zhou, A new procedure for the prediction of the cutting forces in peripheral milling. International Journal of Advanced Manufacturing Technology, (2016) 1-7 online-first, doi=10.1007/s00170-016-9186$\mathrm{Z}$

[38] G. M. Hulbert, Time Finite Element Methods for Structural Dynamics, Int. J. Num. Meth. in Eng. (1992) 33, pp. 307-331

[39] E. Budak, Y. Altintas, Analytical prediction of chatter stability conditions for multidegree of systems in milling, Transactions of ASME Journal of Dynamic Systems Measurement and Control, (1998) 120, pp. 22-30

[40] P. V. Bayly, J. E. Halley, B. P. Mann, Davies, M. A. Stability of interrupted cutting by temporal finite element analysis. Journal of Manufacturing Science and Engineering, (2003) 125(2), 220-225 
[41] O. Elbeyli, J. Q. Sun, On the semi-discretization method for feedback control design of linear systems with time delay. J. Sound Vib. (2004) 273:429-440

[42] F. Hartung, T. Insperger, G. Stepan, J. Turi, Approximate stability charts for milling processes using semi-discretization. Appl. Math. Comput. (2006) 174:51-73

[43] C. Henninger, P. Eberhard, Improving the computational efficiency and accuracy of the semi-discretization method for periodic delay-differential equations. Eur. J. Mech. A-Solid (2008) 27:975-985

[44] G. Floquet, Sur les équations différentielles linéaires à coefficients périodiques. Annales de l'École Normale Supérieure (1883) 12:47-88

[45] C. Henninger, P. Eberhard, Improving the computational efficiency and accuracy of the semi-discretization method for periodic delay-differential equations. European Journal of Mechanics A/Solids 27 (2008) 975-985

[46] R. Szalai, G. Stepan, Lobes and lenses in the stability chart of interrupted turning. Journal of Computational and Nonlinear Dynamics, Transactions of the ASME, (2006) 205-211

[47] Z. Li , Z. Yang, Y. Peng, F. Zhu, X. Ming, Prediction of chatter stability for milling process using Runge-Kutta-based complete discretization method. International Journal of Advanced Manufacturing Technology, (2015) 1-10 online-first, doi $=10.1007 / \mathrm{s} 00170-015-8207-7$

[48] A. Parsian, M. Magnevall, M. Eynian, T. Beno, Time domain simulation of chatter vibrations in indexable drills. International Journal of Advanced Manufacturing Technology, (2016) 1-16 online-first, doi=10.1007/s00170-016-9137-8

[49] Z. Dombovari, G. Stepan, On the bistable zone of milling processes. Philosophical Transactions of the Royal Society A 373 (2015) 20140409

[50] T.G. Molnár, T. Insperger, S.J. Hogan, G. Stepan, Estimation of the bistable zone for machining operations for the case of a distributed cutting-force model. Journal of Computational and Nonlinear Dynamics, (2016) 11(5) 051008

[51] K. Weinert, T. Surmann, D. Enk, O. Webber, The effect of runout on the milling tool vibration and surface quality. Production Engineering - Research and Development. (2007) 1(3), pp. 265-270

[52] M. Krüger, B. Denkena, Model-based identification of tool runout in end milling and estimation of surface roughness from measured cutting forces. The International Journal of Advanced Manufacturing Technology (2013) 65(8) pp. 1067-1080

[53] T.L. Schmitz, J. Couey, E. Marsh, N. Mauntler, D. Hughes, Runout effects in milling: Surface finish, surface location error, and stability. International Journal of Machine Tools and Manufacture (2007) 47(5) pp. 841-851

[54] T. Insperger, B. P. Mann, T. Surmann, G. Stepan, On the chatter frequencies of milling processes with runout, Int. J. Mach. Tools Manuf. (2008) 48, pp. 1081-1089 
